Proceedings of the Edinburgh Mathematical Society (2006) 49, 575-591 (C)

DOI:10.1017/S0013091504001130 Printed in the United Kingdom

\title{
A COMPARISON OF ALGEBRAS OF FUNCTIONS OF BOUNDED VARIATION
}

\author{
BRENDEN ASHTON ${ }^{1}$ AND IAN DOUST ${ }^{2}$ \\ ${ }^{1}$ Canon Information Systems Research Australia, 3 Thomas Holt Drive, \\ North Ryde, NSW 2113, Australia (brenden.ashton@cisra.canon.com.au) \\ ${ }^{2}$ School of Mathematics, University of New South Wales, \\ Sydney, NSW 2052, Australia (i.doust@unsw.edu.au)
}

(Received 29 September 2004)

\begin{abstract}
Motivated by problems in the spectral theory of linear operators, we previously introduced a new concept of variation for functions defined on a non-empty compact subset of the plane. In this paper, we examine the algebras of functions of bounded variation one obtains from these new definitions for the case where the underlying compact set is either a rectangle or the unit circle, and compare these algebras with those previously used by Berkson and Gillespie in their theories of AC-operators and trigonometrically well-bounded operators.
\end{abstract}

Keywords: functions of bounded variation; absolutely continuous functions; functional calculus; well-bounded operators; AC-operators

2000 Mathematics subject classification: Primary 47B40; 26B30

\section{Introduction}

For a function whose domain is a subset of the plane, there are a number of different ways of measuring its variation. For applications in operator theory, one is typically interested in looking at algebras of functions which are defined on the spectrum of some bounded operator. This leads one to seek a definition which is applicable when the domain is a compact subset of the plane.

In modelling operators that possess spectral expansions of a conditional nature, Smart $[\mathbf{1 0}]$ introduced well-bounded operators, which are those operators that admit a functional calculus for the absolutely continuous functions on some compact interval of the real line. Well-bounded operators can be thought of as a generalization to Banach spaces of self-adjoint operators on a Hilbert space. The analogue of unitary operators in this context, the trigonometrically well-bounded operators, were introduced by Berkson and Gillespie [5] using the natural definition for functions of bounded variation on the unit circle. The challenge in providing a suitable analogue of general normal operators was to find a suitable concept of variation for functions defined on an arbitrary compact plane set. In [4], Berkson and Gillespie used a notion of variation due to Hardy [8] and Krause $[\mathbf{9}]$ to introduce an algebra $\operatorname{BV}_{\mathrm{HK}}(J \times K)$ of function of bounded variation 
on rectangles in the plane, and the corresponding notion of an AC-operator. Motivated by a desire to extend the theory of well-bounded and trigonometrically well-bounded operators, Ashton and Doust [3] recently introduced a new definition of variation for complex-valued functions defined on arbitrary compact subsets $\sigma \subset \mathbb{C}$. This definition leads to a Banach algebra BV $(\sigma)$, and a corresponding new class of operators.

It is natural therefore to ask what the relationship is between these earlier concepts of variation and the one introduced in $[\mathbf{3}]$. We shall show in this paper that

(i) $\mathrm{BV}_{\mathrm{HK}}(J \times K) \subset \mathrm{BV}(J \times K)$;

(ii) the inclusion map $\mathrm{BV}_{\mathrm{HK}}(J \times K) \hookrightarrow \mathrm{BV}(J \times K)$ is continuous;

(iii) if $J$ and $K$ are non-degenerate, then $\mathrm{BV}_{\mathrm{HK}}(J \times K) \neq \mathrm{BV}(J \times K)$.

We also show that, for the case where $\sigma$ is the unit circle $\mathbb{T}$, the new definition essentially reproduces the more classical one. We discuss the operator theoretic consequences of these results in $\S 6$.

\section{2. $\mathrm{BV}(\sigma)$ for $\sigma \subset \mathbb{C}$ compact}

Here we shall recall briefly the definition of a function of bounded variation introduced in $[\mathbf{1}]$ and $[\mathbf{3}]$. The reader is referred to $[\mathbf{3}]$ for the full details.

By a curve in the plane we shall mean an element of the set $\Gamma=C([0,1])$. If $\gamma_{1}, \gamma_{2} \in \Gamma$ and if there exists $h:[0,1] \rightarrow[0,1]$, where $h$ is a continuous non-decreasing or nonincreasing surjective function such that $\gamma_{1}(t)=\gamma_{2}(h(t))$ for all $t \in[0,1]$, then we write $\gamma_{1} \cong \gamma_{2}$.

Let $\gamma \in \Gamma$. Then $t \in[0,1]$ is said to be an entry point for $\gamma$ on a line $l$ if either

(i) $t=0$ and $\gamma(0) \in l$, or

(ii) $\gamma(t) \in l$ and for all $\varepsilon>0$ there exists $s \in(t-\varepsilon, t) \cap[0,1]$ such that $\gamma(s) \notin l$.

Suppose that $\gamma \in \Gamma$. We define $\operatorname{vf}(\gamma, l)$ to be the number of entry points of $\gamma$ on $l$.

We set $\operatorname{vf}(\gamma)$ to be the supremum of $\operatorname{vf}(\gamma, l)$ over all lines $l$. We write $\operatorname{vf}_{\mathrm{H}}(\gamma)$ for the supremum of $\operatorname{vf}(\gamma, l)$ over all horizontal lines $l$, and $\mathrm{vf}_{\mathrm{V}}$ for the supremum of $\mathrm{vf}(\gamma, l)$ over all vertical lines. Clearly, $\mathrm{vf} \geqslant \mathrm{vf}_{\mathrm{H}}$ and $\mathrm{vf} \geqslant \mathrm{vf}_{\mathrm{V}}$. We write $\rho$ for $1 / \mathrm{vf}$. If, for example, $\operatorname{vf}(\gamma)=\infty$, then we take the convention that $\rho(\gamma)=0$.

For a set of curves $\left\{\gamma_{j}\right\}_{j=1}^{m} \subset \Gamma$ we define

$$
\operatorname{vf}\left(\bigcup_{j=1}^{m} \gamma_{j}, l\right)=\sum_{j=1}^{m} \operatorname{vf}\left(\gamma_{j}, l\right) \text { and } \operatorname{vf}\left(\bigcup_{j=1}^{m} \gamma_{j}\right)=\sup _{l} \operatorname{vf}\left(\bigcup_{j=1}^{m} \gamma_{j}, l\right) .
$$

The definitions for $\mathrm{vf}_{\mathrm{H}}, \mathrm{vf}_{\mathrm{V}}$ and $\rho$ extend analogously.

Let $\sigma \subset \mathbb{C}$ be compact and let $l$ be a line parametrized by $\mathbb{R}$. Then $t \in \mathbb{R}$ is said to be an entry point of $l$ on $\sigma$ if $l(t) \in \sigma$ and, for all $\varepsilon>0$, there exists $s \in(t-\varepsilon, t)$ such that $l(s) \notin \sigma$. Again set $\operatorname{vf}(\sigma, l)$ to be the number of entry points of $l$ on $\sigma$ and $\operatorname{vf}(\sigma)$ to be 
the supremum of $\operatorname{vf}(\sigma, l)$ over all lines $l$. Clearly, $\operatorname{vf}(\sigma, l)$ does not depend on the choice of parametrization of the line $l$.

Let $z_{0}, \ldots, z_{n} \in \mathbb{C}$. Write $\Pi\left(z_{0}, z_{1}, \ldots, z_{n}\right)$ for the (uniform speed) parametrization of the curve consisting of line segments joining the points $z_{0}, z_{1}, \ldots z_{n}$. We write

$$
\Gamma_{L}=\left\{\gamma \in \Gamma: \gamma \cong \Pi\left(z_{0}, \ldots, z_{n}\right) \text { for some } z_{0}, \ldots z_{n} \in \mathbb{C}\right\}
$$

for the set of all piecewise linear curves.

We shall denote by $\Lambda([0,1])$ the set of all partitions $S=\left\{s_{0}<s_{1}<\cdots s_{n}\right\} \subset[0,1]$. Given $\gamma \in \Gamma$ and $S \in \Lambda([0,1])$, set

$$
\gamma_{S}=\Pi\left(\gamma\left(s_{1}\right), \gamma\left(s_{2}\right), \ldots, \gamma\left(s_{n}\right)\right) \in \Gamma_{L} .
$$

The piecewise linear curve $\gamma_{S}$ is said to be the $S$ approximation of $\gamma$.

Lemma 2.1. Let $\gamma \in \Gamma$ and suppose $\operatorname{vf}(\gamma)<\infty$. Then $\lim _{S \in \Lambda([0,1])} \rho\left(\gamma_{S}\right)=\rho(\gamma)$.

Let $\gamma \in \Gamma$ and let $\emptyset \neq \sigma \subset \mathbb{C}$ be compact. We say that $\left\{z_{i}\right\}_{i=1}^{n}$ is a partition of $\gamma$ over $\sigma$ if $z_{i} \in \sigma$ for all $i$ and if there exists $\left\{s_{0}<s_{1}<\cdots s_{n}\right\} \subset[0,1]$ such that $z_{i}=\gamma\left(s_{i}\right)$ for all $i$. Let $\Lambda(\sigma, \gamma)$ be the lattice of partitions of $\gamma$ over $\sigma$.

Let $f: \sigma \mapsto \mathbb{C}$ and let $\gamma \in \Gamma$. We define the variation along the curve $\gamma$ by

$$
\operatorname{cvar}(f, \gamma, \sigma)=\operatorname{cvar}(f, \gamma)=\sup _{\left\{z_{i}\right\}_{i=1}^{n} \in \Lambda(\sigma, \gamma)} \sum_{i=1}^{n-1}\left|f\left(z_{i+1}\right)-f\left(z_{i}\right)\right| .
$$

Lemma 2.2. Let $f: \sigma \rightarrow \mathbb{C}$. Let $\gamma_{1}, \gamma_{2} \in \Gamma$ and suppose that $\gamma_{1} \cong \gamma_{2}$. Then we have $\operatorname{cvar}\left(f, \gamma_{1}\right)=\operatorname{cvar}\left(f, \gamma_{2}\right)$.

Definition 2.3. Let $f: \sigma \rightarrow \mathbb{C}$. Then variation of $f$ on $\sigma$ is defined to be

$$
\operatorname{var}(f, \sigma)=\sup _{\gamma \in \Gamma} \rho(\gamma) \operatorname{cvar}(f, \gamma)
$$

Here we take the convention that if $\gamma \in \Gamma$ is such that $\rho(\gamma)=0$ and if $\operatorname{cvar}(f, \gamma)=\infty$, then $\rho(\gamma) \operatorname{cvar}(f, \gamma)=0$.

In practice, $\Gamma$ is usually too large a set to work with. As the next lemma shows, one can replace $\Gamma$ by $\Gamma_{L}$.

Lemma 2.4. Let $f: \sigma \rightarrow \mathbb{C}$. Then

$$
\sup _{\gamma \in \Gamma_{L}} \rho(\gamma) \operatorname{cvar}(f, \gamma)=\sup _{\gamma \in \Gamma} \rho(\gamma) \operatorname{cvar}(f, \gamma)
$$

Note that, for subintervals of $\mathbb{R}$, this new definition agrees with the standard one.

Proposition 2.5. Let $f \in \mathrm{BV}([0,1])$. Then

$$
\operatorname{var}_{[0,1]} f=\sup _{\gamma \in \Gamma} \rho(\gamma) \operatorname{cvar}(f, \gamma)
$$

For $f: \sigma \rightarrow \mathbb{C}$, set $\|f\|_{\mathrm{BV}(\sigma)}=\|f\|_{\infty}+\operatorname{var}(f, \sigma)$. The functions of bounded variation with domain $\sigma$ are defined to be

$$
\mathrm{BV}(\sigma)=\left\{f: \sigma \mapsto \mathbb{C}:\|f\|_{\mathrm{BV}(\sigma)}<\infty\right\} .
$$

Theorem 2.6. $\left(\mathrm{BV}(\sigma),\|\cdot\|_{\mathrm{BV}(\sigma)}\right)$ is a Banach algebra. 


\section{A comparison of $\mathrm{BV}(J \times K)$ and $\mathrm{BV}_{\mathrm{HK}}(J \times K)$}

Let $J \times K=[a, b] \times[c, d] \subset \mathbb{R}^{2} \cong \mathbb{C}$ be a fixed rectangle. For functions on such sets, definitions of bounded variation and absolute continuity were given by Hardy and Krause, and these definitions were used by Berkson and Gillespie [4] to define AC operators. The functions of bounded variation in the Hardy-Krause sense form a Banach algebra which we denote $\mathrm{BV}_{\mathrm{HK}}(J \times K)$. Both $\mathrm{BV}(J \times K)$ and $\mathrm{BV}_{\mathrm{HK}}(J \times K)$ are isomorphic to $\mathrm{BV}(J)$ if $K$ is degenerate (that is $K=[c, c]$ ). It is natural to ask about the relationship between $\mathrm{BV}(J \times K)$ and $\mathrm{BV}_{\mathrm{HK}}(J \times K)$, since this will determine how the operator theory based around these new function algebras compares with the established theory. In this section we shall show that $\mathrm{BV}_{\mathrm{HK}}(J \times K)$ forms a subset of $\mathrm{BV}(J \times K)$ and that the inclusion is proper if $J$ and $K$ are non-degenerate. The operator theoretic consequences of this are discussed in $\S 6$.

We say that $\left\{s_{i}, t_{j}\right\}_{i, j=1}^{n, m}$ is a partition of $J \times K$ if $\left\{s_{i}\right\}_{i=1}^{n} \in \Lambda(J)$ and $\left\{t_{j}\right\}_{j=1}^{m} \in \Lambda(K)$. The set of partitions of $J \times K$ is denoted $\Lambda(J \times K)$. Let

$$
S=\left\{s_{i}, t_{j}\right\}_{i, j=1}^{n, m} \in \Lambda(J \times K), \quad T=\left\{s_{i}^{\prime}, t_{j}^{\prime}\right\}_{i, j=1}^{n^{\prime}, m^{\prime}} \in \Lambda(J \times K) .
$$

Then $T$ is said to be a refinement of $S$ if $\left\{s_{i}^{\prime}\right\}_{i=1}^{n^{\prime}}$ is a refinement of $\left\{s_{i}\right\}_{i=1}^{n}$ and $\left\{t_{j}^{\prime}\right\}_{j=1}^{m^{\prime}}$ is a refinement of $\left\{t_{j}\right\}_{j=1}^{m}$. We then write $S \leqslant T$. We shall write $S \vee T$ for the partition with the least number of elements which is a refinement of both $S$ and $T$.

Let $f: J \times K \rightarrow \mathbb{C}$ and let $S=\left\{s_{i}, t_{j}\right\}_{i, j}^{n, m} \in \Lambda(J \times K)$. Define

$$
\omega(f, S)=\sum_{i, j=1}^{n-1, m-1}\left|f\left(s_{i}, t_{j}\right)-f\left(s_{i+1}, t_{j}\right)+f\left(s_{i+1}, t_{j+1}\right)-f\left(s_{i}, t_{j+1}\right)\right| .
$$

The two-dimensional variation in the Hardy-Krause sense is defined as

$$
\operatorname{var}_{\mathrm{HK}}(f, J \times K)=\sup _{S \in \Lambda(J \times K)} \omega(f, S) .
$$

The norm

$$
\|f\|_{\mathrm{BV}_{\mathrm{HK}}}=|f(a, c)|+\operatorname{var}(f(\cdot, c), J)+\operatorname{var}(f(a, \cdot), K)+\operatorname{var}_{\mathrm{HK}}(f, J \times K)
$$

is equivalent to that introduced by Berkson and Gillespie [4]. The functions of bounded variation in the Hardy-Krause sense are defined to be

$$
\operatorname{BV}_{\mathrm{HK}}(J \times K)=\left\{f: J \times K \rightarrow \mathbb{C}:\|f\|_{\mathrm{BV}_{\mathrm{HK}}}<\infty\right\} .
$$

For properties of this Banach algebra see $[4,7]$.

It is an important fact that if $f \in \mathrm{BV}(J)$ is real valued, then there exist $f_{1}, f_{2} \in \mathrm{BV}(J)$ such that $f=f_{1}-f_{2}, f_{1}$ and $f_{2}$ are non-decreasing functions,

$$
\left\|f_{1}\right\|_{\mathrm{BV}(J)} \leqslant\|f\|_{\mathrm{BV}(J)} \quad \text { and } \quad\left\|f_{2}\right\|_{\mathrm{BV}(J)} \leqslant\|f\|_{\mathrm{BV}(J)} .
$$

One of our aims in this section is to show an analogous result for $f \in \mathrm{BV}_{\mathrm{HK}}(J \times K)$. 
Functions of bounded variation on the intervals $J$ and $K$ extend in a natural way to functions of bounded variation on the rectangle $J \times K$. Given $f: J \rightarrow \mathbb{C}$ and $g: K \rightarrow \mathbb{C}$, define $u(f), v(g): J \times K \rightarrow \mathbb{C}$ by $u(f)(x, y)=f(x)$ and $v(g)(x, y)=g(y)$. It is easy to see that if $f \in \mathrm{BV}(J)$ and $g \in \mathrm{BV}(K)$, then $u(f), v(g) \in \mathrm{BV}_{\mathrm{HK}}(J \times K)$ with

$$
\|f\|_{\mathrm{BV}(J)}=\|u(f)\|_{\mathrm{BV}_{\mathrm{HK}}} \quad \text { and } \quad\|g\|_{\mathrm{BV}(K)}=\|v(g)\|_{\mathrm{BV}_{\mathrm{HK}}} .
$$

Let $x \in \mathbb{R}$. We define $x^{+}=\max \{0, x\}$ and $x^{-}=\min \{0, x\}$. Let $f \in \mathrm{BV}_{\mathrm{HK}}(J \times K)$ be real valued. Set

$$
\omega^{+}(f, S)=\sum_{i, j=1}^{n-1, m-1}\left(f\left(s_{i}, t_{j}\right)-f\left(s_{i+1}, t_{j}\right)+f\left(s_{i+1}, t_{j+1}\right)-f\left(s_{i}, t_{j+1}\right)\right)^{+}
$$

and

$$
\omega^{-}(f, S)=-\sum_{i, j=1}^{n-1, m-1}\left(f\left(s_{i}, t_{j}\right)-f\left(s_{i+1}, t_{j}\right)+f\left(s_{i+1}, t_{j+1}\right)-f\left(s_{i}, t_{j+1}\right)\right)^{-} .
$$

Clearly, $\omega(f, S)=\omega^{+}(f, S)+\omega^{-}(f, S)$. For $(x, y) \in J \times K$ set

$$
\begin{aligned}
& v_{f}^{+}(x, y)=\sup _{S \in \Lambda([a, x] \times[c, y])} \omega^{+}(f, S), \\
& v_{f}^{-}(x, y)=\sup _{S \in \Lambda([a, x] \times[c, y])} \omega^{-}(f, S) .
\end{aligned}
$$

The following result is immediate.

Lemma 3.1. Let $f \in \mathrm{BV}_{\mathrm{HK}}(J \times K)$ be real valued. Then $v_{f}^{+}(\cdot, c)=v_{f}^{-}(\cdot, c)=0$ and $v_{f}^{+}(a, \cdot)=v_{f}^{-}(a, \cdot)=0$.

Lemma 3.2. Let $f \in \mathrm{BV}_{\mathrm{HK}}(J \times K)$ be real valued. Fix $x \in J$ and fix $y \in K$. Then the functions $v_{f}^{+}(x, \cdot), v_{f}^{-}(x, \cdot), v_{f}^{+}(\cdot, y)$ and $v_{f}^{-}(\cdot, y)$ are non-decreasing.

Proof. Let $a \leqslant x_{1} \leqslant x_{2} \leqslant b$. Then $\Lambda\left(\left[a, x_{1}\right] \times[c, y]\right) \subset \Lambda\left(\left[a, x_{2}\right] \times[c, y]\right)$. Hence,

$$
\begin{aligned}
v_{f}^{+}\left(x_{1}, y\right) & =\sup _{S \in \Lambda\left(\left[a, x_{1}\right] \times[c, y]\right)} \omega^{+}(f, S) \\
& \leqslant \sup _{S \in \Lambda\left(\left[a, x_{2}\right] \times[c, y]\right)} \omega^{+}(f, S) \\
& =v_{f}^{+}\left(x_{2}, y\right) .
\end{aligned}
$$

The other claims have similar proofs.

Lemma 3.3. Let $f \in \mathrm{BV}_{\mathrm{HK}}(J \times K)$ be real valued and let $(x, y) \in J \times K$. Then

$$
v_{f}^{+}(x, y)+v_{f}^{-}(x, y)=\operatorname{var}_{\mathrm{HK}}(f,[a, x] \times[c, y]) .
$$


Proof.

$$
\begin{aligned}
\operatorname{var}_{\mathrm{HK}}(f,[a, x] \times[c, y]) & =\sup _{S \in \Lambda([a, x] \times[c, y])} \omega(f, S) \\
& =\sup _{S \in \Lambda([a, x] \times[c, y])}\left(\omega^{+}(f, S)+\omega^{-}(f, S)\right) \\
& \leqslant \sup _{S \in \Lambda([a, x] \times[c, y])} \omega^{+}(f, S)+\sup _{S \in \Lambda([a, x] \times[c, y])} \omega^{-}(f, S) \\
& =v_{f}^{+}(x, y)+v_{f}^{-}(x, y) .
\end{aligned}
$$

Fix $\varepsilon>0$. There exist $S_{1}, S_{2} \in \Lambda([a, x] \times[c, y])$ such that $v_{f}^{+}(x, y) \leqslant \omega^{+}\left(f, S_{1}\right)+\frac{1}{2} \varepsilon$ and $v_{f}^{-}(x, y) \leqslant \omega^{-}\left(f, S_{2}\right)+\frac{1}{2} \varepsilon$. Then

$$
\begin{aligned}
v_{f}^{+}(x, y)+v_{f}^{-}(x, y) & \leqslant \omega^{+}\left(f, S_{1}\right)+\omega^{-}\left(f, S_{2}\right)+\varepsilon \\
& \leqslant \omega^{+}\left(f, S_{1} \vee S_{2}\right)+\omega^{-}\left(f, S_{1} \vee S_{2}\right)+\varepsilon \\
& =\omega\left(f, S_{1} \vee S_{2}\right)+\varepsilon \\
& \leqslant \operatorname{var}_{\mathrm{HK}}(f,[a, x] \times[c, y])+\varepsilon .
\end{aligned}
$$

Lemma 3.4. Let $f \in \mathrm{BV}_{\mathrm{HK}}(J \times K)$ be real valued and let $(x, y) \in J \times K$. Then

$$
v_{f}^{+}(x, y)-v_{f}^{-}(x, y)=f(a, c)-f(x, c)+f(x, y)-f(a, y) .
$$

Proof. Fix $\varepsilon>0$. There exists $S \in \Lambda([a, x] \times[c, y])$ such that

$$
\omega^{+}(f, S) \leqslant v_{f}^{+}(x, y) \leqslant \omega^{+}(f, S)+\varepsilon
$$

and

$$
\omega^{-}(f, S) \leqslant v_{f}^{-}(x, y) \leqslant \omega^{-}(f, S)+\varepsilon .
$$

Without loss of generality we can assume that $(a, c),(x, y) \in S$. But, by cancellation of terms, $\omega^{+}(f, S)-\omega^{-}(f, S)=f(a, c)-f(x, c)+f(x, y)-f(a, y)$, and so the result follows.

Lemma 3.5. Let $f \in \mathrm{BV}_{\mathrm{HK}}(J \times K)$ be real valued. Furthermore, suppose for all $\left(x_{1}, y_{1}\right),\left(x_{2}, y_{2}\right) \in J \times K$, where $x_{1} \leqslant x_{2}$ and $y_{1} \leqslant y_{2}$, that $f\left(x_{2}, y_{2}\right)-f\left(x_{1}, y_{2}\right) \geqslant$ $f\left(x_{2}, y_{1}\right)-f\left(x_{1}, y_{1}\right)$. Then, for any $\left[x_{1}^{\prime}, x_{2}^{\prime}\right] \times\left[y_{1}^{\prime}, y_{2}^{\prime}\right] \subset J \times K$,

$$
\operatorname{var}_{\mathrm{HK}}\left(f,\left[x_{1}^{\prime}, x_{2}^{\prime}\right] \times\left[y_{1}^{\prime}, y_{2}^{\prime}\right]\right)=f\left(x_{1}^{\prime}, y_{1}^{\prime}\right)-f\left(x_{2}^{\prime}, y_{1}^{\prime}\right)+f\left(x_{2}^{\prime}, y_{2}^{\prime}\right)-f\left(x_{1}^{\prime}, y_{2}^{\prime}\right) .
$$

Proof. Let

$$
S=\left\{s_{i}, t_{j}\right\}_{i, j=1}^{n, m} \subset \Lambda\left(\left[x_{1}^{\prime}, x_{2}^{\prime}\right] \times\left[y_{1}^{\prime}, y_{2}^{\prime}\right]\right) .
$$

By refining if necessary we can assume that $\left(s_{1}, t_{1}\right)=\left(x_{1}^{\prime}, y_{1}^{\prime}\right)$ and $\left(s_{n}, t_{m}\right)=\left(x_{2}^{\prime}, y_{2}^{\prime}\right)$. By assumption, for each $i$ and $j, f\left(s_{i}, t_{j}\right)-f\left(s_{i+1}, t_{j}\right)+f\left(s_{i+1}, t_{j+1}\right)-f\left(s_{i}, t_{j+1}\right) \geqslant 0$. Hence, $\omega(f, S)=\omega^{+}(f, S)$. There is therefore cancellation of terms in $\omega(f, S)$, giving

$$
\omega(f, S)=f\left(x_{1}^{\prime}, y_{1}^{\prime}\right)-f\left(x_{2}^{\prime}, y_{1}^{\prime}\right)+f\left(x_{2}^{\prime}, y_{2}^{\prime}\right)-f\left(x_{1}^{\prime}, y_{2}^{\prime}\right) .
$$

Taking the supremum over all $S \in \Lambda(J \times K)$ on the left-hand side gives the result. 
Lemma 3.6. Let $f \in \mathrm{BV}_{\mathrm{HK}}(J \times K)$ be real valued and let $\left[x_{1}, x_{2}\right] \times\left[y_{1}, y_{2}\right] \subset J \times K$. Then

$$
\operatorname{var}_{\mathrm{HK}}\left(v_{f}^{+},\left[x_{1}, x_{2}\right] \times\left[y_{1}, y_{2}\right]\right)=v_{f}^{+}\left(x_{1}, y_{1}\right)-v_{f}^{+}\left(x_{2}, y_{1}\right)+v_{f}^{+}\left(x_{2}, y_{2}\right)-v_{f}^{+}\left(x_{1}, y_{2}\right)
$$

and

$$
\operatorname{var}_{\mathrm{HK}}\left(v_{f}^{-},\left[x_{1}, x_{2}\right] \times\left[y_{1}, y_{2}\right]\right)=v_{f}^{-}\left(x_{1}, y_{1}\right)-v_{f}^{-}\left(x_{2}, y_{1}\right)+v_{f}^{-}\left(x_{2}, y_{2}\right)-v_{f}^{-}\left(x_{1}, y_{2}\right) .
$$

Proof. To prove the first equality it suffices to show, by Lemma 3.5, that, for all $\left(x_{1}, y_{1}\right),\left(x_{2}, y_{2}\right) \in J \times K$, where $x_{1} \leqslant x_{2}$ and $y_{1} \leqslant y_{2}$,

$$
v_{f}^{+}\left(x_{2}, y_{2}\right)-v_{f}^{+}\left(x_{1}, y_{2}\right) \geqslant v_{f}^{+}\left(x_{2}, y_{1}\right)-v_{f}^{+}\left(x_{1}, y_{1}\right) \text {. }
$$

For $i, j \in\{1,2\}$ let $S_{i, j} \in \Lambda\left(\left[a, x_{i}\right] \times\left[c, y_{j}\right]\right)$ be such that $S_{1,1} \leqslant S_{1,2} \leqslant S_{2,2}$ and $S_{1,1} \leqslant$ $S_{2,1} \leqslant S_{2,2}$. Then cancellation gives $\omega^{+}\left(f, S_{2,2}\right)-\omega^{+}\left(f, S_{1,2}\right) \geqslant \omega^{+}\left(f, S_{2,1}\right)-\omega^{+}\left(f, S_{1,1}\right)$. The result now follows by refining $S_{2,2}$ (and hence all $S_{i, j}$ ). The second equality has a similar proof.

Lemma 3.7. Let $f \in \mathrm{BV}_{\mathrm{HK}}(J \times K)$ be real valued. Then

$$
\operatorname{var}_{\mathrm{HK}}\left(v_{f}^{+}, J \times K\right) \leqslant \operatorname{var}_{\mathrm{HK}}(f, J \times K)
$$

and

$$
\operatorname{var}_{\mathrm{HK}}\left(v_{f}^{-}, J \times K\right) \leqslant \operatorname{var}_{\mathrm{HK}}(f, J \times K) .
$$

Proof. This follows immediately from Lemmas 3.1, 3.3 and 3.6.

Let $f \in \mathrm{BV}_{\mathrm{HK}}(J \times K)$ be real valued. Then $f$ is said to have property (UR) (or 'up and to the right') if it satisfies the following conditions:

(i) for each $x \in[a, b]$ the function $f(x, \cdot): K \rightarrow \mathbb{R}$ is non-decreasing;

(ii) for each $y \in[c, d]$ the function $f(\cdot, y): J \rightarrow \mathbb{R}$ is non-decreasing;

(iii) $\operatorname{var}_{\mathrm{HK}}\left(f,\left(x_{1}, x_{2}\right) \times\left(y_{1}, y_{2}\right)\right)=f\left(x_{1}, y_{1}\right)-f\left(x_{1}, y_{2}\right)+f\left(x_{2}, y_{2}\right)-f\left(x_{2}, y_{1}\right)$ for all $\left(x_{1}, x_{2}\right) \times\left(y_{1}, y_{2}\right) \subset J \times K$.

Similarly, we say that $f$ has property (UL) if 'non-decreasing' is replaced by 'non-increasing' in (ii). Our aim is to show that if $f \in \mathrm{BV}_{\mathrm{HK}}(J \times K)$ is real valued, then $f=g-h$, where $g$ and $h$ are (UR), $\|g\|_{\mathrm{BV}_{\mathrm{HK}}} \leqslant\|f\|_{\mathrm{BV}_{\mathrm{HK}}}$ and $\|h\|_{\mathrm{BV}_{\mathrm{HK}}} \leqslant\|f\|_{\mathrm{BV}_{\mathrm{HK}}}$.

Let $l=\Pi\left(z_{1}, z_{2}\right)$. Then $l$ is said to be (UR) if either

(i) $\operatorname{Re}\left(z_{1}\right) \leqslant \operatorname{Re}\left(z_{2}\right)$ and $\operatorname{Im}\left(z_{1}\right) \leqslant \operatorname{Im}\left(z_{2}\right)$ or

(ii) $\operatorname{Re}\left(z_{1}\right) \geqslant \operatorname{Re}\left(z_{2}\right)$ and $\operatorname{Im}\left(z_{1}\right) \geqslant \operatorname{Im}\left(z_{2}\right)$.

Similarly, $l$ is said to be (UL) if either

(i) $\operatorname{Re}\left(z_{1}\right) \leqslant \operatorname{Re}\left(z_{2}\right)$ and $\operatorname{Im}\left(z_{1}\right) \geqslant \operatorname{Im}\left(z_{2}\right)$ or

(ii) $\operatorname{Re}\left(z_{1}\right) \geqslant \operatorname{Re}\left(z_{2}\right)$ and $\operatorname{Im}\left(z_{1}\right) \leqslant \operatorname{Im}\left(z_{2}\right)$. 
Line segments parametrized by $[0,1]$ are either (UL) or (UR), or both. If $\gamma \in \Gamma_{L}$, then we can write $\gamma \cong l_{1} \circ l_{2} \circ \cdots \circ l_{n}$, where each $l_{i}$ is a line segment parametrized by $[0,1]$. Then $\gamma$ is said to be (UL) (respectively, (UR)) if each $l_{i}$ is (UL) (respectively, (UR)). Clearly, if $\gamma_{1}, \gamma_{2} \in \Gamma_{L}$ and $\gamma_{1} \cong \gamma_{2}$, then, for example, $\gamma_{1}$ is (UR) if and only if $\gamma_{2}$ is (UR). Also, for example, if $\gamma_{1}, \gamma_{2} \in \Gamma_{L}$ are both (UL), then so is their concatenation $\gamma_{1} \circ \gamma_{2}$.

Lemma 3.8. Let $f \in \mathrm{BV}_{\mathrm{HK}}(J \times K)$ be real valued. Then $v_{f}^{+}$and $v_{f}^{-}$have property $(U R)$.

Proof. This follows from Lemmas 3.2 and 3.6.

Lemma 3.9. Let $f \in \mathrm{BV}_{\mathrm{HK}}(J \times K)$ be real valued and let $g \in \mathrm{BV}(J)$. Suppose that $f$ has property $(U R)$ and $g$ is non-decreasing. $f+u(g)$ then has property $(U R)$.

Proof. Clearly, $f+u(g)$ has properties (i) and (ii). To see that it has property (iii) we note, for any $\left(x_{1}^{\prime}, y_{1}^{\prime}\right),\left(x_{2}^{\prime}, y_{2}^{\prime}\right) \in J \times K$, that

$u(g)\left(x_{1}^{\prime}, y_{1}^{\prime}\right)-u(g)\left(x_{2}^{\prime}, y_{1}^{\prime}\right)+u(g)\left(x_{2}^{\prime}, y_{2}^{\prime}\right)-u(g)\left(x_{1}^{\prime}, y_{2}^{\prime}\right)=g\left(x_{1}^{\prime}\right)-g\left(x_{2}^{\prime}\right)+g\left(x_{2}^{\prime}\right)-g\left(x_{1}^{\prime}\right)=0$,

and so

$$
\begin{aligned}
(f+u(g))\left(x_{1}^{\prime}, y_{1}^{\prime}\right)-(f+u(g))\left(x_{2}^{\prime}, y_{1}^{\prime}\right)+ & (f+u(g))\left(x_{2}^{\prime}, y_{2}^{\prime}\right)-(f+u(g))\left(x_{1}^{\prime}, y_{2}^{\prime}\right) \\
& =f\left(x_{1}^{\prime}, y_{1}^{\prime}\right)-f\left(x_{2}^{\prime}, y_{1}^{\prime}\right)+f\left(x_{2}^{\prime}, y_{2}^{\prime}\right)-f\left(x_{1}^{\prime}, y_{2}^{\prime}\right) .
\end{aligned}
$$

Hence, if $\left[x_{1}, x_{2}\right] \times\left[y_{1}, y_{2}\right] \subset J \times K$, then $\omega(f+u(g), S)=\omega(f, S)$ for all $S \in \Lambda\left(\left[x_{1}, x_{2}\right] \times\right.$ $\left.\left[y_{1}, y_{2}\right]\right)$. So $\operatorname{var}_{H K}\left(f+u(g),\left[x_{1}, x_{2}\right] \times\left[y_{1}, y_{2}\right]\right)=\operatorname{var}_{H K}\left(f,\left[x_{1}, x_{2}\right] \times\left[y_{1}, y_{2}\right]\right)$. Then

$$
\begin{aligned}
\operatorname{var}_{\mathrm{HK}} & \left(f+u(g),\left[x_{1}, x_{2}\right] \times\left[y_{1}, y_{2}\right]\right) \\
\quad & =\operatorname{var}_{\mathrm{HK}}\left(f,\left[x_{1}, x_{2}\right] \times\left[y_{1}, y_{2}\right]\right) \\
& =f\left(x_{1}, y_{1}\right)-f\left(x_{2}, y_{1}\right)+f\left(x_{2}, y_{2}\right)-f\left(x_{1}, y_{2}\right) \\
& =(f+u(g))\left(x_{1}, y_{1}\right)-(f+u(g))\left(x_{2}, y_{1}\right)+(f+u(g))\left(x_{2}, y_{2}\right)-(f+u(g))\left(x_{1}, y_{2}\right) .
\end{aligned}
$$

Remark 3.10. The previous lemma also holds if we take $g \in \mathrm{BV}(K), g$ nondecreasing, and use $v$ instead of $u$.

Proposition 3.11. Let $f \in \mathrm{BV}_{\mathrm{HK}}(J \times K)$ be real valued. There then exist $g, h \in$ $\mathrm{BV}_{\mathrm{HK}}(J \times K)$ such that

(i) $f=g-h$,

(ii) $g$ and $h$ are $(U R)$, and

(iii) $\|g\|_{\mathrm{BV}_{\mathrm{HK}}} \leqslant\|f\|_{\mathrm{BV}_{\mathrm{HK}}}$ and $\|h\|_{\mathrm{BV}_{\mathrm{HK}}} \leqslant\|f\|_{\mathrm{BV}_{\mathrm{HK}}}$. 
Proof. There exist $f_{1}, f_{2} \in \mathrm{BV}(J)$ such that $f(\cdot, c)-f(a, c)=f_{1}-f_{2}, f_{1}, f_{2}$ are non-decreasing, $\operatorname{var}\left(f_{1}, J\right) \leqslant \operatorname{var}(f(\cdot, c), J)$ and $\operatorname{var}\left(f_{2}, J\right) \leqslant \operatorname{var}(f(\cdot, c), J)$. There also exist $f_{3}, f_{4} \in \mathrm{BV}(K)$ such that $f(a, \cdot)-f(a, c)=f_{3}-f_{4}, f_{3}, f_{4}$ are non-decreasing, $\operatorname{var}\left(f_{3}, K\right) \leqslant \operatorname{var}(f(a, \cdot), K)$ and $\operatorname{var}\left(f_{4}, K\right) \leqslant \operatorname{var}(f(a, \cdot), K)$. Set $g=v_{f}^{+}+u\left(f_{1}\right)+v\left(f_{3}\right)+$ $f(a, c)$ and $h=v_{f}^{-}+u\left(f_{2}\right)+v\left(f_{4}\right)$. Then, by Lemmas 3.8 and 3.9, $g$ and $h$ are (UR). By Lemma 3.4,

$$
\begin{aligned}
f(x, y)= & v_{f}^{+}(x, y)-v_{f}^{-}(x, y)+f(x, c)+f(a, y)-f(a, c) \\
= & v_{f}^{+}(x, y)-v_{f}^{-}(x, y)+\left(f_{1}(x)-f_{2}(x)+f(a, c)\right) \\
& \quad+\left(f_{3}(y)-f_{4}(y)+f(a, c)\right)-f(a, c) \\
= & v_{f}^{+}(x, y)+f_{1}(x)+f_{3}(y)+f(a, c)-\left(v_{f}^{-}(x, y)+f_{2}(x)+f_{4}(y)\right) \\
= & g(x, y)-h(x, y) .
\end{aligned}
$$

Also, using Lemma 3.7,

$$
\begin{aligned}
\|g\|_{\mathrm{BV}} & =|g(a, c)|+\operatorname{var}(g(\cdot, c), J)+\operatorname{var}(g(a, \cdot), K)+\operatorname{var}_{\mathrm{HK}}(g, J \times K) \\
& =|f(a, b)|+\operatorname{var}\left(f_{1}, J\right)+\operatorname{var}\left(f_{3}, K\right)+\operatorname{var}_{\mathrm{HK}}\left(v_{f}^{+}, J \times K\right) \\
& \leqslant|f(a, b)|+\operatorname{var}(f(\cdot, c), J)+\operatorname{var}(f(a, \cdot), K)+\operatorname{var}_{\mathrm{HK}}(f, J \times K) \\
& =\|f\|_{\mathrm{BV}} .
\end{aligned}
$$

Similarly, $\|h\|_{\mathrm{BV}_{\mathrm{HK}}} \leqslant\|f\|_{\mathrm{BV}_{\mathrm{HK}}}$.

Remark 3.12. Note that, similarly, we can write $f=g-h$, where $g$ and $h$ have the same norm inequalities but are (UL) instead of (UR).

Lemma 3.13. Let $f \in \mathrm{BV}_{\mathrm{HK}}(J \times K)$ satisfy property $(U R)$. Let $\left\{h_{j}\right\}_{j=1}^{m} \subset \Gamma_{L}$ be a set of horizontal line segments. Suppose that $n=\operatorname{vf}_{\mathrm{V}}\left(\bigcup_{j=1}^{m} h_{j}\right)$. Then

$$
\frac{1}{n} \sum_{j=1}^{m} \operatorname{cvar}\left(f, h_{j}\right) \leqslant\|f\|_{\mathrm{BV}_{\mathrm{HK}}} .
$$

Proof. Recall that $J \times K=[a, b] \times[c, d]$. The idea of the proof is to replace each line segment $h_{j}$ by another horizontal line segment $h_{j}^{\prime}$, where the image of each $h_{j}^{\prime}$ is a subset of $J \times\{d\}$, and to then show the inequality for the $h_{j}^{\prime}$. For $1 \leqslant j \leqslant m$, let $\left(x_{j}, y_{j}\right)$ and $\left(x_{j}^{\prime}, y_{j}\right)$ be the left and right endpoints of $h_{j}$. Let $h_{j}^{\prime}=\Pi\left(\left(x_{j}, d\right),\left(x_{j}^{\prime}, d\right)\right)$ for each $j$. Since $f$ is (UR), whenever $x_{j} \leqslant s \leqslant t \leqslant x_{j}^{\prime}$, we have $f\left(t, y_{j}\right)-f\left(s, y_{j}\right) \leqslant f(t, d)-$ $f(s, d)$ and hence $\left|f\left(t, y_{j}\right)-f\left(s, y_{j}\right)\right| \leqslant|f(t, d)-f(s, d)|$. From this we can conclude that $\operatorname{cvar}\left(f, h_{j}\right) \leqslant \operatorname{cvar}\left(f, h_{j}^{\prime}\right)$ for all $j$. Also $\operatorname{vf}_{\mathrm{V}}\left(\bigcup_{j=1}^{m} h_{j}\right)=\operatorname{vf}_{\mathrm{V}}\left(\bigcup_{j=1}^{m} h_{j}^{\prime}\right)$. Using the same proof as in Proposition 2.5 we can conclude that

$$
\rho_{\mathrm{V}}\left(\bigcup_{j=1}^{m} h_{j}^{\prime}\right) \sum_{j=1}^{m} \operatorname{cvar}\left(f, h_{j}^{\prime}\right) \leqslant \operatorname{cvar}(f, \Pi((a, d),(b, d))) .
$$


Hence,

$$
\begin{aligned}
\frac{1}{n} \sum_{j=1}^{m} \operatorname{cvar}\left(f, h_{j}\right) & \leqslant \frac{1}{n} \sum_{j=1}^{m} \operatorname{cvar}\left(f, h_{j}^{\prime}\right) \\
& \leqslant \operatorname{cvar}(f, \Pi((a, d),(b, d))) \\
& =\operatorname{var}(f(\cdot, d), J) \\
& \leqslant\|f\|_{\mathrm{BV}_{\mathrm{HK}}} .
\end{aligned}
$$

The penultimate step follows by the monotonicity of $f(\cdot, d)$.

Remark 3.14. A similar lemma holds when $\left\{l_{i}\right\}_{i=1}^{m}$ are vertical line segments and/or $f$ has property (UL).

Lemma 3.15. Let $f \in \mathrm{BV}_{\mathrm{HK}}(J \times K)$ satisfy property $(U R)$. Let $\left\{\gamma_{j}\right\}_{j=1}^{m} \subset \Gamma_{L}$ be such that $\gamma_{j}$ is $(U R)$ for all $j$. Then

$$
\rho\left(\bigcup_{j=1}^{m} \gamma_{j}\right) \sum_{j=1}^{m} \operatorname{cvar}\left(f, \gamma_{j}\right) \leqslant 2\|f\|_{\mathrm{BV}_{\mathrm{HK}}} .
$$

Proof. For each $j$ we shall replace $\gamma_{j}$ by horizontal and vertical lines. Using complex number notation, set

$$
h_{j}=\Pi\left(\gamma_{j}(0), \operatorname{Re}\left(\gamma_{j}(1)\right)+\mathrm{i} \operatorname{Im}\left(\gamma_{j}(0)\right)\right)
$$

and

$$
v_{j}=\Pi\left(\operatorname{Re}\left(\gamma_{j}(1)\right)+\mathrm{i} \operatorname{Im}\left(\gamma_{j}(0)\right), \gamma_{j}(1)\right) .
$$

Since $f$ is (UR) then $\operatorname{cvar}\left(f, \gamma_{j}\right)=\operatorname{cvar}\left(f, h_{j}\right)+\operatorname{cvar}\left(f, v_{j}\right)$. Also note that, for any vertical line $l, \operatorname{vf}\left(\bigcup_{j=1}^{m} \gamma_{j}, l\right)=\operatorname{vf}\left(\bigcup_{j=1}^{m} h_{j}, l\right)$ and so

$$
\rho\left(\bigcup_{j=1}^{m} \gamma_{j}\right) \leqslant \rho_{\mathrm{V}}\left(\bigcup_{j=1}^{m} \gamma_{j}\right)=\rho_{\mathrm{V}}\left(\bigcup_{j=1}^{m} h_{j}\right) .
$$

Similarly, $\rho\left(\bigcup_{j=1}^{m} \gamma_{j}\right) \leqslant \rho_{\mathrm{H}}\left(\bigcup_{j=1}^{m} v_{j}\right)$. Hence,

$$
\begin{aligned}
\rho\left(\bigcup_{j=1}^{m} \gamma_{j}\right) \sum_{j=1}^{m} \operatorname{cvar}\left(f, \gamma_{j}\right) & =\rho\left(\bigcup_{j=1}^{m} \gamma_{j}\right) \sum_{j=1}^{m}\left(\operatorname{cvar}\left(f, h_{j}\right)+\operatorname{cvar}\left(f, v_{j}\right)\right) \\
& \leqslant \rho_{\mathrm{V}}\left(\bigcup_{j=1}^{m} h_{j}\right) \sum_{j=1}^{m} \operatorname{cvar}\left(f, h_{j}\right)+\rho_{\mathrm{H}}\left(\bigcup_{j=1}^{m} v_{j}\right) \sum_{j=1}^{m} \operatorname{cvar}\left(f, v_{j}\right) \\
& \leqslant 2\|f\|_{\mathrm{BV}_{\mathrm{HK}}} .
\end{aligned}
$$

The last step follows from Lemma 3.13 .

Theorem 3.16. The inclusion $\mathrm{BV}_{\mathrm{HK}}(J \times K) \hookrightarrow \mathrm{BV}(J \times K)$ is continuous. 
Proof. It is sufficient to show that if $f \in \operatorname{BV}_{\mathrm{HK}}(J \times K)$ is real valued, then we have

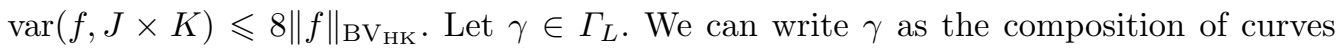
which alternate between being (UL) and (UR). Without loss of generality assume that $\gamma \cong \lambda_{1} \circ \mu_{1} \circ \lambda_{2} \circ \mu_{2} \circ \cdots \circ \lambda_{n} \circ \mu_{n}$, where each $\lambda_{i} \in \Gamma_{L}$ is (UL) and each $\mu_{i}$ is (UR). By Lemma 2.2, $\operatorname{cvar}(f, \gamma)=\sum_{i=1}^{n}\left(\operatorname{cvar}\left(f, \lambda_{i}\right)+\operatorname{cvar}\left(f, \mu_{i}\right)\right)$. By Proposition 3.11 we can write $f=f_{1}-g_{1}=f_{2}-g_{2}$, where $f_{1}$ and $g_{1}$ satisfy property (UR) and $f_{2}$ and $g_{2}$ satisfy (UL). Then $\operatorname{vf}\left(\bigcup_{i=1}^{n} \lambda_{i}\right) \leqslant \operatorname{vf}(\gamma)$ and so $\rho\left(\bigcup_{i=1}^{n} \lambda_{i}\right) \geqslant \rho(\lambda)$. Similarly, $\rho\left(\bigcup_{i=1}^{n} \mu_{i}\right) \geqslant \rho(\gamma)$. Using Lemma 3.15 and Proposition 3.11 we have

$$
\begin{aligned}
\rho(\gamma) \operatorname{cvar}(f, \gamma)= & \rho(\gamma) \sum_{i=1}^{n}\left(\operatorname{cvar}\left(f, \lambda_{i}\right)+\operatorname{cvar}\left(f, \mu_{i}\right)\right) \\
\leqslant & \rho(\gamma) \sum_{i=1}^{n}\left(\operatorname{cvar}\left(f_{1}, \lambda_{i}\right)+\operatorname{cvar}\left(g_{1}, \lambda_{i}\right)+\operatorname{cvar}\left(f_{2}, \mu_{i}\right)+\operatorname{cvar}\left(g_{2}, \mu_{i}\right)\right) \\
\leqslant & \rho\left(\bigcup_{i=1}^{n} \lambda_{i}\right)\left(\sum_{i=1}^{n} \operatorname{cvar}\left(f_{1}, \lambda_{i}\right)+\sum_{i=1}^{n} \operatorname{cvar}\left(g_{1}, \lambda_{i}\right)\right) \\
& \quad+\rho\left(\bigcup_{i=1}^{n} \mu_{i}\right)\left(\sum_{i=1}^{n} \operatorname{cvar}\left(f_{2}, \mu_{i}\right)+\sum_{i=1}^{n} \operatorname{cvar}\left(g_{2}, \mu_{i}\right)\right) \\
\leqslant & 2\left\|f_{1}\right\|_{\mathrm{BV}_{\mathrm{HK}}}+2\left\|g_{1}\right\|_{\mathrm{BV}}+2\left\|f_{2}\right\|_{\mathrm{BV}}+2\left\|g_{2}\right\|_{\mathrm{BV}_{\mathrm{HK}}} \\
\leqslant & 4 \times 2\|f\|_{\mathrm{BV}} \mathrm{HK} \\
= & 8\|f\|_{\mathrm{BV}_{\mathrm{HK}}} .
\end{aligned}
$$

The result now follows from Lemma 2.4.

Example 3.17. Here we show that if $J \times K$ is non-degenerate, then $\operatorname{BV}_{\mathrm{HK}}(J \times K) \subsetneq$ $\mathrm{BV}(J \times K)$. Without loss of generality, assume that $J \times K$ is the unit square. Let $A \subset$ $J \times K$ be the closed triangle with vertices at 0,1 and $1+i$. We show that $\chi_{A} \in \operatorname{BV}(J \times K)$ but $\chi_{A} \notin \mathrm{BV}_{\mathrm{HK}}(J \times K)$.

It is easy to see that if $B$ is a half-plane, then $\operatorname{var}\left(\chi_{B}, J \times K\right) \leqslant 1$ and so $\chi_{B} \in$ $\mathrm{BV}(J \times K)$. In particular $B=\{x+\mathrm{i} y \subset \mathbb{C}: x \leqslant y\}$ is a half-plane and so $\chi_{A}=\chi_{B} \mid J \times$ $K \in \mathrm{BV}(J \times K)$.

Fix $n \in \mathbb{N}$. Set $t_{i}=i / n$. Then

$$
S=\left\{t_{i}, t_{j}\right\}_{i, j=0}^{n, n} \in \Lambda(J \times K) .
$$

For each $i, \chi_{A}\left(t_{i}, t_{i}\right)-\chi_{A}\left(t_{i+1}, t_{i}\right)+\chi_{A}\left(t_{i+1}, t_{i+1}\right)-\chi_{A}\left(t_{i}, t_{i+1}\right)=1-0+1-1=1$. Hence,

$$
\omega\left(\chi_{A}, S\right) \geqslant \sum_{i=0}^{n-1}\left|\chi_{A}\left(t_{i}, t_{i}\right)-\chi_{A}\left(t_{i+1}, t_{i}\right)+\chi_{A}\left(t_{i+1}, t_{i+1}\right)-\chi_{A}\left(t_{i}, t_{i+1}\right)\right|=n .
$$

So $\operatorname{var}_{\mathrm{HK}}\left(\chi_{A}, J \times K\right) \geqslant n$. Hence, $\chi_{A} \notin \mathrm{BV}_{\mathrm{HK}}(J \times K)$. 


\section{4. $\mathrm{AC}(\sigma)$ for $\sigma \subset \mathbb{C}$ compact}

From an operator theoretic point of view, one would like to be able to deduce structural information about an operator $T$ from bounds on $\|p(T)\|$ for $p$ in some small algebra of functions. Let $\mathcal{P}$ denote the set of all complex polynomials. In the case that $X$ is reflexive and $\sigma(T) \subset \mathbb{R}$, then a bound of the form $\|p(T)\| \leqslant C\|p\|_{C(\sigma(T))}$ for all $p \in \mathcal{P}$ is sufficient to show that $T$ can be written as an integral with respect to a countably additive spectral measure, whereas a weaker bound of the form $\|p(T)\| \leqslant C\left\|_{B V}\right\|_{B V[a, b]}$ for $p \in \mathcal{P}$ implies that $T$ has an integral representation with respect to a spectral family of projections. If the spectrum is not real, then one would not expect to be able to prove much unless the algebra contains at least $\mathcal{P}_{2}$, the polynomials in two variables (or equivalently the polynomials in $z$ and $\bar{z}$ ). This leads to our definition of the absolutely continuous functions defined on a non-empty compact subset $\sigma$ of $\mathbb{C}$.

Definition 4.1. $\mathrm{AC}(\sigma)$, the set of absolutely continuous functions on $\sigma$, is defined to be the closure in $\mathrm{BV}(\sigma)$ of $\mathcal{P}_{2}$.

Note that, by [3, Corollary 3.14], the polynomials are always of bounded variation. For any non-empty compact subset $\sigma, \mathrm{AC}(\sigma)$ is a Banach subalgebra of $\mathrm{BV}(\sigma)$. If $\sigma=$ $[a, b] \subset \mathbb{R}$, then $\mathrm{AC}(\sigma)$ coincides with the usual notion of absolute continuity.

We shall now compare our definition of $\mathrm{AC}(J \times K)$ with the definition used by Berkson and Gillespie [4], which corresponds to the Hardy-Krause definition of variation. Let $\mu$ be the Lebesgue measure on $\mathbb{C}$ and $f: J \times K \rightarrow \mathbb{C}$. We say that $f$ is absolutely continuous in the Hardy-Krause sense if, for each $\varepsilon>0$, there is a $\delta>0$ such that for any finite collection of rectangles $\left\{R_{i}\right\}_{i=1}^{n}$ whose sides are parallel to the axes and whose interiors are disjoint and such that $\sum_{i=1}^{n} \mu\left(R_{i}\right)<\delta$, then $\sum_{i=1}^{n} \operatorname{var}_{H K}\left(f, R_{i}\right)<\varepsilon$. We denote the set of such functions by $\mathrm{AC}_{\mathrm{HK}}(J \times K)$. The set $\mathrm{AC}_{\mathrm{HK}}(J \times K)$ forms a Banach subalgebra of $\mathrm{BV}_{\mathrm{HK}}(J \times K)$, and is the closure of $\mathcal{P}_{2}$ in the $\mathrm{BV}_{\mathrm{HK}}(J \times K)$ norm (see [4]). If $K$ is degenerate, then $\mathrm{BV}_{\mathrm{HK}}(J \times K)$ reduces to the standard algebra $\mathrm{AC}(J)$. (See $[\mathbf{4}, \mathbf{7}]$ for properties of $\operatorname{AC}_{\mathrm{HK}}(J \times K)$.) The following result is an immediate consequence of Theorem 3.16 and the density of the polynomials in both algebras.

Theorem 4.2. The inclusion $\mathrm{AC}_{\mathrm{HK}}(J \times K) \hookrightarrow \mathrm{AC}(J \times K)$ is continuous.

Remark 4.3. If $J \times K$ is non-degenerate, then $\operatorname{AC}_{\mathrm{HK}}(J \times K) \subsetneq \mathrm{AC}(J \times K)$. For example, let $J \times K=[0,1] \times[0,1]$. Let $f$ be the function $J \times K \rightarrow \mathbb{R}$ defined by

$$
f(x, y)= \begin{cases}0 & \text { if } x<y \\ x-y & \text { if } x \geqslant y\end{cases}
$$

Then $f$ is continuous and piecewise planar and so, by [3, Lemma 4.10], $f \in \operatorname{AC}(J \times K)$. For $i, n \in \mathbb{N}$, where $i \leqslant n$, set

$$
R_{i, n}=\left[\frac{i-1}{n}, \frac{i}{n}\right] \times\left[\frac{i-1}{n}, \frac{i}{n}\right] .
$$

Then

$$
\lim _{n \rightarrow \infty} \sum_{i=1}^{n} \mu\left(R_{i, n}\right)=\lim _{n \rightarrow \infty} n \times \frac{1}{n^{2}}=0 .
$$


But

$$
\begin{aligned}
& \lim _{n \rightarrow \infty} \sum_{i=1}^{n} \operatorname{var}_{\mathrm{HK}}\left(f, R_{i, n}\right) \\
& \quad \geqslant \lim _{n \rightarrow \infty} \sum_{i=1}^{n}\left|f\left(\frac{i-1}{n}, \frac{i-1}{n}\right)-f\left(\frac{i}{n}, \frac{i-1}{n}\right)+f\left(\frac{i}{n}, \frac{i}{n}\right)-f\left(\frac{i-1}{n}, \frac{i}{n}\right)\right| \\
& \quad=\lim _{n \rightarrow \infty} \sum_{i=1}^{n}\left|0-\frac{1}{n}+0-0\right| . \\
& \quad=1 .
\end{aligned}
$$

Hence, $f \notin \mathrm{AC}_{\mathrm{HK}}(J \times K)$.

\section{A comparison of $B V_{\text {new }}(\mathbb{T})$ and $B V_{\text {old }}(\mathbb{T})$}

In [5] Berkson and Gillespie introduced the class of trigonometrically well-bounded operators. These operators, which have formed an important tool in their study of operator-valued harmonic analysis, are defined as operators that admit a weakly compact functional calculus for the absolutely continuous functions on the unit circle in $\mathbb{C}$ (or, equivalently, are of the form $\mathrm{e}^{\mathrm{i} A}$, where $A$ is well bounded of type (B)). The concepts of variation and absolute continuity used in $[5]$ are just the natural extensions obtained by transferring the usual definitions for an interval in $\mathbb{R}$ onto the unit circle. In this section we show that our new definitions are equivalent to these earlier ones used in this setting.

For $f: \mathbb{T} \rightarrow \mathbb{C}$, let $\operatorname{var}_{\mathbb{T}}(f)=\operatorname{var}_{[0,2 \pi]} f\left(\mathrm{e}^{\mathrm{i}(\cdot)}\right)$. That is

$$
\operatorname{var}_{\mathbb{T}}(f)=\sup _{\mathcal{P}} \sum_{j=1}^{n}\left|f\left(\omega_{j}\right)-f\left(\omega_{j-1}\right)\right|,
$$

where the supremum is taken over all partitions $\mathcal{P}=\left\{\omega_{j}=\mathrm{e}^{\mathrm{i} \theta_{j}}\right\}_{j=1}^{n}$ of the circle with $0=\theta_{0} \leqslant \theta_{1} \leqslant \cdots \leqslant \theta_{n}=2 \pi$. Berkson and Gillespie worked with the following norm and Banach algebra:

$$
\begin{aligned}
\|f\|_{\mathrm{BV}_{\text {old }}} & =\sup _{z \in \mathbb{T}}|f|+\operatorname{var}_{\mathbb{T}}(f) \\
\mathrm{BV}_{\text {old }}(\mathbb{T}) & =\left\{f: \mathbb{T} \rightarrow \mathbb{C}:\|f\|_{\mathrm{BV}_{\text {old }}}<\infty\right\}
\end{aligned}
$$

For comparison, we shall write $\mathrm{BV}_{\text {new }}(\mathbb{T})$ for the algebra given by the definitions in $\S 2$. The subalgebras obtained by taking the closures of the trigonometric polynomials in these algebras will be denoted by $\mathrm{AC}_{\text {old }}(\mathbb{T})$ and $\mathrm{AC}_{\text {new }}(\mathbb{T})$.

Lemma 5.1. For all $f: \mathbb{T} \rightarrow \mathbb{C}$,

$$
\operatorname{var}_{\mathbb{T}}(f) \leqslant 2 \operatorname{var}(f, \mathbb{T})
$$


Proof. Let $\mathcal{P}=\left\{\omega_{j}\right\}_{j=1}^{n}$ be a partition of $\mathbb{T}$. Let $\gamma_{\mathcal{P}}=\Pi\left(\omega_{0}, \ldots, \omega_{n}\right)$. Then

$$
\operatorname{cvar}\left(f, \gamma_{\mathcal{P}}\right)=\sum_{j=1}^{n}\left|f\left(\omega_{j}\right)-f\left(\omega_{j-1}\right)\right|
$$

and $\rho\left(\gamma_{\mathcal{P}}\right)=\frac{1}{2}$. Thus,

$$
\begin{aligned}
\operatorname{var}_{\mathbb{T}}(f) & =\sup _{\mathcal{P}} \operatorname{cvar}\left(f, \gamma_{\mathcal{P}}\right) \\
& =2 \sup _{\mathcal{P}} \rho\left(\gamma_{\mathcal{P}}\right) \operatorname{cvar}\left(f, \gamma_{\mathcal{P}}\right) \\
& \leqslant 2 \sup _{\gamma \in \Gamma} \rho(\gamma) \operatorname{cvar}(f, \gamma) \\
& =2 \operatorname{var}(f, \mathbb{T}) .
\end{aligned}
$$

We note that the value of 2 is sharp in this inequality since, if $f$ is the characteristic function of a single point in $\mathbb{T}$, then $\operatorname{var}_{\mathbb{T}}(f)=2$ and $\operatorname{var}(f, \mathbb{T})=1$.

Before proving a reverse inequality, we introduce some notation and terminology.

Definition 5.2. A reparametrization of $\mathbb{T}$ is any continuous, orientation preserving bijection $\tau: \mathbb{T} \rightarrow \mathbb{T}$.

Definition 5.3. Let $\Gamma_{L, \mathbb{T}}=\left\{\Pi\left(z_{0}, \ldots, z_{n}\right): z_{0}, \ldots, z_{n} \in \mathbb{T}\right\}$ denote the set of piecewise linear curves with vertices on $\mathbb{T}$.

Given $f: \mathbb{T} \rightarrow \mathbb{C}$ and any reparametrization $\tau$ of $\mathbb{T}$, let $f_{\tau}=f \circ \tau$. Clearly, $\operatorname{var}_{\mathbb{T}}(f)=$ $\operatorname{var}_{\mathbb{T}}\left(f_{\tau}\right)$. The reparametrization $\tau$ also determines a map $\Gamma_{L, \mathbb{T}} \rightarrow \Gamma_{L, \mathbb{T}}$,

$$
\gamma=\Pi\left(z_{0}, \ldots, z_{n}\right) \mapsto \gamma_{\tau}=\Pi\left(\tau\left(z_{0}\right), \ldots, \tau\left(z_{n}\right)\right) .
$$

Lemma 5.4. Suppose that $f$ and $\tau$ are as above and that $\gamma \in \Gamma_{L, \mathbb{T}}$. Then

(i) $\operatorname{vf}(\gamma)=\operatorname{vf}\left(\gamma_{\tau}\right)$,

(ii) $\rho(\gamma) \operatorname{cvar}(f, \gamma)=\rho\left(\gamma_{\tau}\right) \operatorname{cvar}\left(f_{\tau}, \gamma_{\tau}\right)$,

(iii) $\operatorname{var}_{\mathbb{T}} f=\operatorname{var}_{\mathbb{T}} f_{\tau}$.

Proof. Part (i) follows immediately from the observation that if the chords $\gamma=$ $\Pi\left(z_{0}, z_{1}\right)$ and $\ell=\Pi\left(w_{0}, w_{1}\right)$ intersect, then so do $\gamma_{\tau}$ and $\ell_{\tau}$. Clearly, $\operatorname{cvar}(f, \gamma)=$ $\operatorname{cvar}\left(f_{\tau}, \gamma_{\tau}\right)$ so this proves (ii). The final statement is obvious.

Theorem 5.5. For all $f \in \mathrm{BV}(\mathbb{T})$,

$$
\operatorname{var}(f, \mathbb{T}) \leqslant \operatorname{var}_{\mathbb{T}}(f) .
$$


Proof. Suppose that $\gamma \in \Gamma_{L}$ intersects $\mathbb{T}$ at $\gamma\left(s_{0}\right), \ldots, \gamma\left(s_{n}\right)$ with $s_{0}<s_{1}<\cdots<s_{n}$. (We may assume that $n \geqslant 1$.) Let $S=\left\{s_{j}\right\}_{j=0}^{n}$ and let $\gamma_{S}=\Pi\left(\gamma\left(s_{0}\right), \gamma\left(s_{n}\right)\right)$ denote the $S$ approximation to $\gamma$. As in the proof of [3, Lemma 3.1], $\rho\left(\gamma_{S}\right) \geqslant \rho(\gamma)$, and so

$$
\rho(\gamma) \operatorname{cvar}(f, \gamma) \leqslant \rho\left(\gamma_{S}\right) \operatorname{cvar}\left(f, \gamma_{S}\right) .
$$

Now choose a reparametrization $\tau$ such that $\operatorname{Im}\left(\tau\left(z_{j}\right)\right)>0$ for $j=1, \ldots, n$. Let $\gamma_{2}=$ $\left(\gamma_{S}\right)_{\tau}$. Then, by Lemma 5.4,

$$
\rho\left(\gamma_{S}\right) \operatorname{cvar}\left(f, \gamma_{S}\right)=\rho\left(\gamma_{2}\right) \operatorname{cvar}\left(f_{\tau}, \gamma_{2}\right) .
$$

Define $f_{3}:[-1,1] \rightarrow \mathbb{C}, f_{3}(t)=f_{\tau}\left(\sqrt{1-t^{2}}\right)$, and $\gamma_{3}=$ Re $\circ \gamma_{2}$. Thus, the image of $\gamma_{3}$ is a subset of $[-1,1]$. Clearly, then

$$
\operatorname{vf}\left(\gamma_{3}\right)=\operatorname{vf}_{\mathrm{V}}\left(\gamma_{3}\right)=\operatorname{vf}_{\mathrm{V}}\left(\gamma_{2}\right) \leqslant \operatorname{vf}\left(\gamma_{2}\right),
$$

and so $\rho\left(\gamma_{2}\right) \leqslant \rho\left(\gamma_{3}\right)$. Note also that $\operatorname{cvar}\left(f_{\tau}, \gamma_{2}\right) \leqslant \operatorname{cvar}\left(f_{3}, \gamma_{3}\right)$. Thus,

$$
\begin{array}{rlrl}
\rho(\gamma) \operatorname{cvar}(f, \gamma) & \leqslant \rho\left(\gamma_{2}\right) \operatorname{cvar}\left(f_{\tau}, \gamma_{2}\right) & & (\text { by }(5.1) \text { and }(5.2)) \\
& \leqslant \rho\left(\gamma_{3}\right) \operatorname{cvar}\left(f_{3}, \gamma_{3}\right) & & \\
& \leqslant \operatorname{var}_{[-1,1]} f_{3} & & (\text { by }[\mathbf{3}, \text { Proposition 3.6]) } \\
& =\operatorname{var}_{[0, \pi]} f_{\tau}\left(\mathrm{e}^{\mathrm{i}(\cdot)}\right) & \\
& \leqslant \operatorname{var}_{\mathbb{T}} f_{\tau} & \\
& =\operatorname{var}_{\mathbb{T}} f & \text { (by Lemma } 5.4) .
\end{array}
$$

Taking the supremum over all $\gamma \in \Gamma_{L}$ gives the result.

Corollary 5.6. The Banach algebras $\mathrm{BV}_{\text {new }}(\mathbb{T})$ and $\mathrm{BV}_{\text {old }}(\mathbb{T})$ are isomorphic.

Corollary 5.7. The Banach algebras $\mathrm{AC}_{\text {new }}(\mathbb{T})$ and $\mathrm{AC}_{\text {old }}(\mathbb{T})$ are isomorphic.

\section{Operator theory}

In this section we shall note some of the operator theoretic consequences of the results in this paper.

We shall say that an operator $T \in B(X)$ is an $\operatorname{AC}(\sigma)$ operator if it admits an $\operatorname{AC}(\sigma)$ functional calculus; that is, if there exists a continuous Banach algebra homomorphism $\Psi: \operatorname{AC}(\sigma) \rightarrow B(X)$ such that $\Psi(1)=I$ and $\Psi(\lambda)=T$ (where, as before, $\lambda$ is the identity function $\lambda(z)=z)$. The theory of $\mathrm{AC}(\sigma)$ operators is pursued more fully in [2]. It is shown there that much of the theory of well-bounded operators generalizes to cover $\mathrm{AC}(\sigma)$ operators. For example, compact $\mathrm{AC}(\sigma)$ operators have conditionally convergent spectral expansions similar to those of well-bounded operators. In another direction, $\mathrm{AC}(\sigma)$ operators on reflexive spaces (or more generally those for which the $\mathrm{AC}(\sigma)$ functional calculus is weakly compact) admit a family of projections that generalizes the spectral family of a well-bounded operator.

Berkson and Gillespie [4] defined an operator to be an AC operator if it admits an $\operatorname{AC}_{\mathrm{HK}}(J \times K)$ functional calculus for some rectangle $J \times K$. It is natural to ask what the relationship is between these two classes of operator. 
Theorem 6.1. If $T \in B(X)$ is an $\mathrm{AC}(\sigma)$ operator, then $T$ is an $\mathrm{AC}$ operator (in the sense of Berkson and Gillespie), and hence there exist commuting well-bounded operators $A, B \in B(X)$ such that $T=A+\mathrm{i} B$.

Proof. Suppose that $T$ is an $\mathrm{AC}(\sigma)$ operator with a functional calculus map $\Psi$ : $\mathrm{AC}(\sigma) \rightarrow B(X)$. Let $J \times K$ be any rectangle containing $\sigma$. Define

$$
\hat{\Psi}: \mathrm{AC}_{\mathrm{HK}}(J \times K) \rightarrow B(X)
$$

by $\hat{\Psi}(f)=\Psi(f \mid \sigma)$. It follows from Theorem 4.2 and [3, Lemma 4.5] that this map is a well-defined algebra homomorphism with

$$
\|\hat{\Psi}(f)\| \leqslant\|\Psi\|\|f \mid \sigma\|_{\mathrm{BV}(\sigma)} \leqslant\|\Psi\|\|f\|_{B V(J \times K)} \leqslant 8\|\Psi\|\|f\|_{\mathrm{BV}_{\mathrm{HK}}(J \times K)} .
$$

It follows that $T$ has an $\operatorname{AC}_{\mathrm{HK}}(J \times K)$ functional calculus.

The splitting into real and imaginary parts is necessarily unique if $X$ is reflexive [4]. There are, however, examples on non-reflexive spaces of AC operators with more than one splitting of this type (see [6, Example 3.1]). Each such splitting gives a different $\operatorname{AC}_{\mathrm{HK}}(J \times K)$ functional calculus. The situation for $\mathrm{AC}(\sigma)$ operators is less clear, however. $\mathrm{An} \mathrm{AC}(\sigma)$ operator may still have more than one representation in the form $A+\mathrm{i} B$, but not all such representations give rise to an $\mathrm{AC}(\sigma)$ functional calculus. In particular, no example is known of an operator with more than one $\mathrm{AC}(\sigma)$ functional calculus.

It is not difficult to see that the class of $\mathrm{AC}(\sigma)$ operators is in fact strictly smaller than the class of AC operators. Lemma 4.1 of [3] shows that if $T \in B(X)$ is an $\mathrm{AC}(\sigma)$ operator, then, for all $\alpha, \beta \in \mathbb{C}, \alpha T+\beta I$ is an $\mathrm{AC}(\alpha \sigma+\beta)$ operator. It follows that the example from $[6]$ of an $\mathrm{AC}$ operator $T$ such that $(1+i) T$ is not an $\mathrm{AC}$ operator also gives an example of an $\mathrm{AC}$ operator that is not an $\mathrm{AC}(\sigma)$ operator (for any $\sigma$ ).

One of the most important subclasses of AC operators has been the family of trigonometrically well-bounded operators. The following is a consequence of Corollary 5.7 and the definition of being trigonometrically well bounded [5].

Theorem 6.2. An operator $T \in B(X)$ is a trigonometrically well-bounded operator if and only if it admits a weakly compact $\mathrm{AC}(\mathbb{T})$ functional calculus. In particular, if $X$ is reflexive, then $T$ is a trigonometrically well-bounded operator if and only if it is an $\mathrm{AC}(\mathbb{T})$ operator.

Acknowledgements. The authors acknowledge the support of an Australian Postgraduate Award (B.A.) and a grant from the Australian Research Council (I.D.).

\section{References}

1. B. Ashton, Functions of bounded variation in two variables and $\mathrm{AC}(\sigma)$ operators, $\mathrm{PhD}$ thesis, University of New South Wales (2000).

2. B. Ashton, $\mathrm{AC}(\sigma)$ operators, preprint.

3. B. Ashton And I. Doust, Functions of bounded variation on compact subsets of the plane, Studia Math. 169 (2005), 163-188. 
4. E. Berkson AND T. A. Gillespie, Absolutely continuous functions of two variables and well-bounded operators, J. Lond. Math. Soc. (2) 30 (1984), 305-321.

5. E. Berkson And T. A. Gillespie, AC functions on the circle and spectral families, $J$. Operat. Theory 13 (1985), 33-47.

6. E. Berkson, I. Doust And T. A. Gillespie, Properties of AC-operators, Acta Sci. Math. (Szeged) 6 (1997), 249-271.

7. J. A. Clarkson and C. R. Adams, On definitions of bounded variation for functions of two variables, Trans. Am. Math. Soc. 35 (1933), 824-854.

8. G. H. HARDY, On double Fourier series, and especially those which represent the double zeta-function with real and incommensurable parameters, Q. J. Pure Appl. Math. 37 (1906), 53-79.

9. M. KRause, Über Fouriersche Reihen mit zwei veränderlichen Grössen, Ber. Verhandl. Sächs. Gesell. Wiss. Leipzig 55 (1903), 164-197.

10. D. R. Smart, Conditionally convergent spectral expansions, J. Austral. Math. Soc. A 1 (1960), 319-333. 